\title{
Carbon star survey in the Local Group
}

\section{Probing the stellar halo of NGC $147^{\star}$}

\author{
P. Battinelli ${ }^{1}$ and S. Demers ${ }^{2}$ \\ 1 INAF, Osservatorio Astronomico di Roma, Viale del Parco Mellini 84, 00136 Roma, Italia \\ 2 Département de Physique, Université de Montréal, C.P.6128, Succursale Centre-Ville, Montréal, Québec, H3C 3J7, Canada \\ e-mail: demers@astro.umontreal.ca
}

Received 27 June 2003 / Accepted 12 January 2004

\begin{abstract}
We have imaged a $42^{\prime} \times 28^{\prime}$ field centered on the spheroidal galaxy NGC 147 to study its AGB population and determine the main structural properties of this M 31 companion. This field was observed through two broadband $(R$ and $I)$ and two narrowband $(\mathrm{CN}$ and $\mathrm{TiO})$ filters, following the standard approach of our systematic survey of $\mathrm{C}$ stars in nearby galaxies. We identified $288 \mathrm{C}$ stars in NGC 147. The average $I$-magnitude of such sample of C stars is $\langle I\rangle=20.31$ with $\sigma=0.40$. If we adopt a distance modulus $\mu_{0}=24.39$, derived from the luminosity of both the tip of the red giant branch (TRGB) and the horizontal branch (HB) by Han et al., we conclude that the average absolute $I$-magnitude of $\mathrm{C}$ stars is $\left\langle M_{I}\right\rangle=-4.39$, thus slightly under luminous with respect to what was found for other galaxies in our survey. From the radial surface profile we derive a scale length of $4.1 \pm 0.1^{\prime}$ and a tidal radius $r_{\mathrm{t}}=33.9 \pm 2.4^{\prime}$, a value quite larger than previous estimates. The $\mathrm{C}$ star population shows a scale length remarkably similar, thus suggesting that intermediate-age stars in NGC 147 are well mixed with the older population. Finally, the overall $C / M$ ratio is $0.24 \pm 0.02$ and its local values increase monotonically from the center up to 3.5 scale lengths (beyond which counts become too low to yield reliable $C / M$ ratios). Such radial behaviour implies a corresponding decrease of about $0.4 \mathrm{dex}$ in $[\mathrm{Fe} / \mathrm{H}]$, confirming a well defined metallicity gradient in NGC 147.
\end{abstract}

Key words. galaxies: individual: NGC 147 - galaxies: stellar content - galaxies: structure

\section{Introduction}

Asymptotic giant branch (AGB) stars are the major contributors to the integral luminosity of intermediate-age systems. Evolutionary models are a challenge because of the difficult treatment of the Thermal Pulsing phase, mass loss and mixing length. In spite of these well known difficulties in modeling the evolution of a complex stellar population, the recent improvements of both evolutionary tracks and the new treatment of the variable molecular opacities during the TP-AGB (Marigo 2003), allowed Marigo et al. (2003) to successfully simulate all the main features shown by the stellar population towards the LMC. Detailed models for carbon Star (C star, hereafter) populations in systems with different metallicities have also been recently obtained by Mouhcine \& Lançon (2003).

On the basis of the nearly equal mean apparent magnitudes of the RR Lyrae variables in both galaxies (Saha et al. 1990;

Send offprint requests to: P. Battinelli,

e-mail: battinel@oarhp1.rm.astro.it

* Based on observations carried on at the Canada-France-Hawaii Telescope, operated by the National Research Council of Canada, the Centre National de la Recherche Scientifique de France, and the University of Hawaii and on observations obtained at the Italian Telescopio Nazionale Galileo.
Saha \& Hoessel 1990), van den Bergh (1998) suggested that NGC 147 and NGC 185 may probably form a bound pair. At a distance of about $660 \mathrm{kpc}$ (van den Bergh 1998), they appear to have a projected separation of barely $11 \mathrm{kpc}$, i.e. less than the distance between the LMC and the SMC. A recent support to this idea has been brought by Salaris \& Cassisi (1998) who found that the TRGB luminosity is essentially equal in both galaxy. Unfortunately, distance determinations for these two galaxies are not free from uncertainties: e.g. while Saha et al. (1990) find as distance to NGC $147 \mu_{0}=23.92$, Salaris \& Cassisi (1998) obtain $\mu_{0}=24.27$.

Detailed analysis of the stellar populations of those galaxies has shown that they differ markedly in many aspects. Contrary to NGC 147, NGC 185 contains gas, dust and a population of blue stars in its center (Martínez-Delgado et al. 1999). NGC 147 shows an evident intermediate-age population (Han et al. 1997) but it appears almost completely dust and gas free (Young \& Lo 1997; Sage et al. 1998; Young 1999). In the lack of knowledge about the kinematics of this pair of galaxies, Sage et al. (1998) found that the time since the last hypothetical interaction of NGC 147 with M 31 is of the same order as the age of the youngest stars (about $1 \mathrm{Gyr}$ ). Furthermore, the chemical composition of NGC 147 and NGC 185 are not identical, their 
mean $[\mathrm{Fe} / \mathrm{H}]$ being respectively -0.9 (Han et al. 1997) and -1.4 (Lee et al. 1993), both shows sign of radial abundance gradient.

This pair of galaxies seems to be perfect as a stellar laboratory to test the theoretical predictions based on the sophisticated models recently developed by Mouhcine (2001) and Mouhcine \& Lançon (2003). These authors predict that the main statistical properties of the $\mathrm{C}$ star populations are "mainly determined by the current metallicity and that star formation history has no significant effect". For instance, the observed correlation between metallicity and $C / M$ ratio is explained by models as a metallicity sequence rather than an age sequence. On the other hand, the luminosity function (LF) or the distribution on the colour-magnitude plane of $\mathrm{C}$ stars is sensitive to both age and metallicity. Another theoretical prediction suggested by Mouhcine \& Lançon (2003) is that the mean absolute bolometric magnitude of $\mathrm{C}$ stars can be used as a standard candle. It is worth to remind however, that Mouhcine \& Lançon's models do not account for the variation in the molecular opacities due to a change of surface abundances of carbon and oxygen in stars that experience the third dredge-up phase (Marigo 2003).

We have shown in recent years, (see e.g. Letarte et al. 2002) that $\mathrm{C}$ stars are admirably suited to trace tenuous outer regions of galaxies. The main reason being that they are seen against a zero foreground: there is no confusion with Galactic stars seen along the line of sight because there are no $\mathrm{C}$ stars in the Galactic halo ${ }^{1}$ and because dwarf $\mathrm{C}$ stars are too few to count. Deep colour-magnitude diagrams (CMD) are not as useful to trace weak structures because of the numerous Galactic M dwarfs polluting the giant branch. Thanks to this approach we discovered an extended halo made of both old and intermediate-age stars surrounding the NGC 6822 dwarf galaxy (Letarte et al. 2002). Such finding represents the first positive detection of an intermediate-age halo. Extended old halos have also been observed in a few dwarf irregular galaxies (Aparicio \& Tikhonov 2000; Aparicio et al. 2000) but their true size has not yet been mapped. This fact suggests that what we "see" of dwarf galaxies corresponds to their bright region of current star formation and that stars were formed in a much larger volume. It is now known that the LMC has a 20 degree diameter (Irwin 1990 or Weinberg \& Nikolaev 2001). The nature of extended halos is still not clear and our very recent finding about NGC 3109 makes this subject even more puzzling: indeed, this galaxy seems to completely lack any appreciable stellar halo (Demers et al. 2003) even though it is a Magellanic dwarf with no massive neighbors exactly like NGC 6822.

So far we do not know how common is the presence of such intermediate-age halos and, in particular, how spheroidal galaxies behave in this regard. NGC 147 and NGC 185 are the nearest and best suited spheroidals to investigate this phenomenon. The larger dwarf ellipticals, NGC 205 and M 32, are too close to $\mathrm{M} 31$ and suffer from heavy contamination by the Andromeda stellar component. In the present

\footnotetext{
1 The few dozen C stars belonging to the Sagittarius stream do not represent a problem when studying the stellar population of a galaxy since they would appear too bright to be confused with genuine $\mathrm{C}$ stars in the target galaxy.
}

paper we describe our CFHT and TNG observations along with the data reduction and photometric calibration in Sect. 2; the colour-magnitude and colour-colour diagrams in Sect. 3; comparison with previously identified $\mathrm{C}$ stars and discussion of results are given in Sects. 4 and 5. The results of NGC 185 observations will be presented in a forthcoming paper of this series.

\section{Observations and calibrations}

Observations, carried on in service mode at the Canada-FranceHawaii Telescope on August 7th and September 4th 2002, consist in Mould $R, I$, and $\mathrm{CN}$ and TiO imaging fields centered on NGC 147. Each pixel of the CFH12K camera corresponds to 0.206 arcsec yielding a total field of view of a $42^{\prime} \times 28^{\prime}$, thus large enough to well encompass the nominal size of this galaxy $\left(12^{\prime} \times 18^{\prime}\right.$, van den Bergh 2000). A summary of observations and seeing (as FWHM of the PSF) conditions is given in Table 1. The instrumental magnitudes are calibrated using the equations provided by the CFHT QSO team based on numerous Landolt (1992) equatorial standards ${ }^{2}$. These equations, for $R$ and $I$ magnitudes are:

$$
\begin{aligned}
& R=26.190+m_{r}-0.09\left(X_{r}-1\right)+0.0094(R-I), \\
& I=26.185+m_{i}-0.04\left(X_{i}-1\right)-0.0511(R-I),
\end{aligned}
$$

where $X_{r}$ and $X_{i}$ are the airmasses of the $R$ and $I$ exposures. In term of instrumental magnitudes $r$ and $i$, obtained with DAOPHOT, and for the given exposure times, $m_{r}$ and $m_{i}$ are given by:

$m_{r}=(r-25.0)+2.5 \log (250)$

$m_{i}=(i-25.0)+2.5 \log (240)$.

By substracting the above $R$ and $I$ equations and using the appropriate airmasses we obtain:

$(R-I)=0.045+1.064(r-i)$

$I=i+7.1298-0.0511(R-I)$.

Data reduction was obtained by using DAOPHOT/ALLSTAR on each image.

The only published $R, I$ observations of NGC 147 are those published by Mould et al. (1983). We cross identified 50 stars and determined the magnitude and colour differences (Mould's magnitudes minus ours) with our data. We obtained $\Delta_{I}=-0.004 \pm 0.006$ and $\Delta_{R-I}=0.002 \pm 0.018$ thus in excellent agreement with the zero points of our photometry.

In order to search for intergalactic $\mathrm{C}$ stars, a region half way between NGC 185 and NGC 147 has been observed with DOLORES (Device Optimized for the LOw RESolution) installed at the Nasmyth B focus of the Telescopio Nazionale Galileo during the night of October 9/10 2002. The detector is a Loral thinned and back-illuminated $2048 \times 2048$ CCD

\footnotetext{
2 For more details see: http://www.cfht.hawaii.edu/Instruments/Elixir/ stds.2003.05.html
} 
Table 1. Summary of the observations

\begin{tabular}{llccc}
\hline \hline Telescope & Filter & exposure & seeing $\left({ }^{\prime \prime}\right)$ & airmass \\
\hline$C F H T$ & $R$ & $250 \mathrm{~s}$ & 0.93 & 1.140 \\
$C F H T$ & $I$ & $240 \mathrm{~s}$ & 0.73 & 1.143 \\
$C F H T$ & $\mathrm{CN}$ & $560 \mathrm{~s}$ & 0.61 & 1.150 \\
$C F H T$ & $\mathrm{TiO}$ & $560 \mathrm{~s}$ & 0.71 & 1.151 \\
$T N G$ & $R$ & $2 \times 450 \mathrm{~s}$ & 1.29 & 1.26 \\
$T N G$ & $I$ & $2 \times 450 \mathrm{~s}$ & 1.10 & 1.31 \\
\hline
\end{tabular}

with a scale of 0.275 arcsec/px corresponding to a field of view of about 9.4 $\times$ 9.4. $R, I, \mathrm{CN}$ and $\mathrm{TiO}$ images were obtained with total exposure of $900 \mathrm{~s}$ for the broad-band filters and $2700 \mathrm{~s}$ for the narrow-band ones. Unfortunately, the presence of clouds during the second part of the night made the $\mathrm{CN}$ and $\mathrm{TiO}$ images too shallow and thus useless for our purposes. Several Landolt (1992) and Stetson ${ }^{3}$ standard stars, observed during both nights, were used for the photometric calibration of our data.

The zero-point of the CFHT narrow band observations is obtained by setting the average $(\mathrm{CN}-\mathrm{TiO})$ of blue stars equal to zero. This method is explained by Letarte et al. (2002). We adopt their definition of blue star as a star in the colour range $0.0<(R-I)_{0}<0.45$. To convert observed $(R-I)$ colours into intrinsic colours we adopt $E(B-V)=0.17$ (Mould et al. 1983) and then we convert it into $E(R-I)$ using the relation given by Battinelli et al. (2003).

\section{Results}

\subsection{The colour-magnitude diagram}

Figure 1 shows the colour-magnitude diagram of 54640 stars observed at the CFHT and for which the photometric error $\sigma_{(R-I)}<0.10$. A vertical ridge at $(R-I) \approx 0.45$ corresponding to the main-sequence turnoff of field $\mathrm{G}$ dwarfs (which intrinsic colour is $(R-I)_{0} \approx 0.35$, Cox 2000), seen at increasing distances along the line of sight, is clearly evident. This is in fair agreement with a reddening toward NGC 147 of $E(R-I)=0.14$ given by van den Bergh (2000). Our photometry goes about two magnitudes fainter than the TRGB located at $I_{\text {TRGB }}=20.55$ (Han et al. 1997) thus deep enough to fully cover the expected luminosity range of $\mathrm{C}$ stars.

\subsection{The colour-colour diagram}

Figure 2 presents the colour-colour diagram of 30791 stars with photometry in all the four bandwidths. Only stars with a combined error $\epsilon=\sqrt{\sigma_{(R-I)}^{2}+\sigma_{(\mathrm{CN}-\mathrm{TiO})}^{2}}$ smaller than $0.125 \mathrm{mag}$ are retained. The two boxes define the regions of O-rich and C-rich stars as explained by Letarte et al. (2002). The total number of $\mathrm{C}$ stars found is 288, this number makes NGC 147 quite normal for its luminosity

\footnotetext{
${ }^{3}$ Stetson's photometry is available at the Canadian Astronomical Data Center's Web site at http://cadcwww.dao.nrc.ca/ astrocat
}

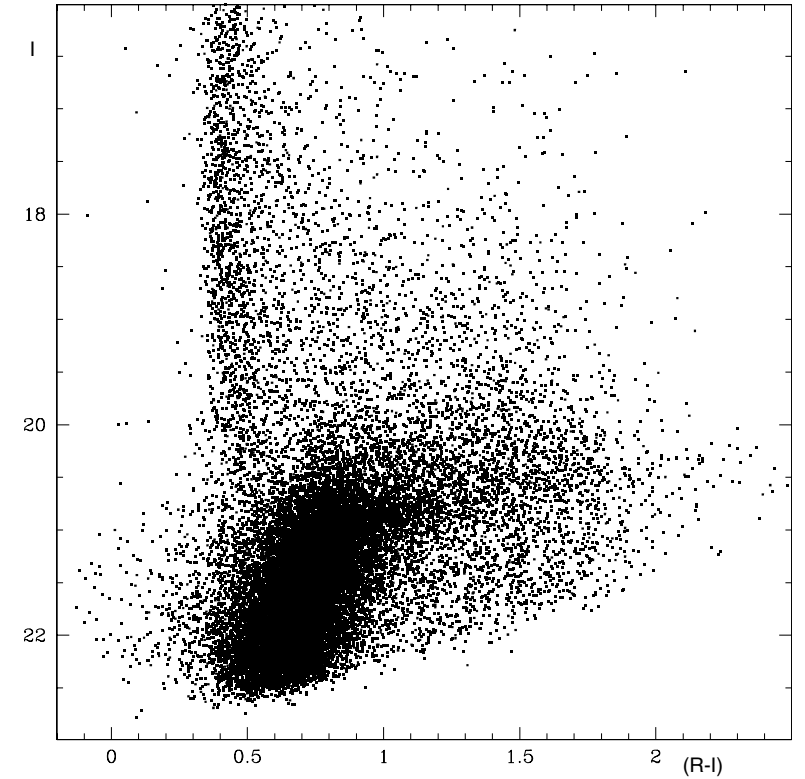

Fig. 1. The colour-magnitude diagram of the $42^{\prime} \times 28^{\prime} \mathrm{CFH} 12 \mathrm{~K}$ field centered on NGC 147 . Only stars with $\sigma_{(R-I)}<0.1$ are plotted.

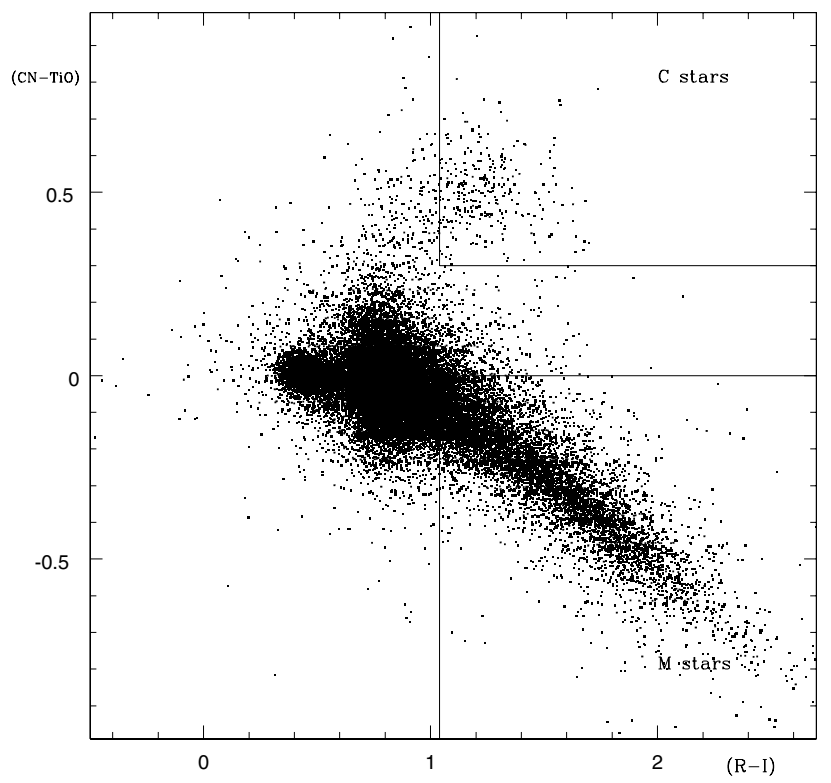

Fig. 2. The colour-colour diagram of same field shown in Fig. 1. Only stars with $\epsilon<0.125$ are plotted (see text). The two boxes define the samples of $\mathrm{C}$ and $\mathrm{M}$ stars.

(Battinelli \& Demers, in preparation). Table 2 lists equatorial coordinates (J2000.0) as well as photometric data for the 288 C stars identified.

\section{Discussion}

\subsection{The $C$ star population}

From the colour-colour plot presented in the previous section we can pick up C-rich and O-rich stars according to their colours. Figure 3 shows the CFH12K mosaic field with C stars highlighted as black squares.

Similarly to what we found for the majority of the galaxies surveyed so far, luminosity function of $\mathrm{C}$ stars is well 
Table 2. C stars in NGC $147^{a}$.

\begin{tabular}{ccccccccc}
\hline \hline id & RA & Dec & $I$ & $\sigma_{I}$ & $R-I$ & $\sigma_{R-I}$ & CN-TiO & $\sigma_{\mathrm{CN}-\mathrm{TiO}}$ \\
\hline 1 & $00: 31: 29.13$ & $48: 17: 28.00$ & 20.650 & 0.019 & 1.481 & 0.040 & 0.337 & 0.056 \\
2 & $00: 31: 31.60$ & $48: 35: 31.80$ & 20.265 & 0.013 & 1.127 & 0.021 & 0.489 & 0.044 \\
3 & $00: 31: 58.03$ & $48: 21: 38.80$ & 20.211 & 0.014 & 1.174 & 0.023 & 0.414 & 0.042 \\
4 & $00: 32: 02.42$ & $48: 39: 23.20$ & 20.668 & 0.019 & 1.244 & 0.033 & 0.352 & 0.068 \\
5 & $00: 32: 09.53$ & $48: 30: 35.80$ & 20.057 & 0.011 & 1.076 & 0.018 & 0.559 & 0.037 \\
6 & $00: 32: 11.93$ & $48: 31: 02.00$ & 19.665 & 0.010 & 1.141 & 0.016 & 0.519 & 0.032 \\
7 & $00: 32: 13.61$ & $48: 26: 58.00$ & 20.442 & 0.015 & 1.185 & 0.028 & 0.500 & 0.037 \\
8 & $00: 32: 16.89$ & $48: 26: 41.70$ & 20.745 & 0.020 & 1.458 & 0.046 & 0.362 & 0.072 \\
9 & $00: 32: 18.10$ & $48: 21: 08.10$ & 20.298 & 0.016 & 1.388 & 0.030 & 0.499 & 0.040 \\
10 & $00: 32: 19.26$ & $48: 22: 31.30$ & 19.960 & 0.015 & 1.271 & 0.022 & 0.511 & 0.039 \\
11 & $00: 32: 20.56$ & $48: 23: 58.20$ & 21.420 & 0.029 & 1.531 & 0.077 & 0.384 & 0.056 \\
12 & $00: 32: 21.48$ & $48: 38: 18.50$ & 20.054 & 0.013 & 1.090 & 0.021 & 0.639 & 0.033 \\
13 & $00: 32: 23.23$ & $48: 18: 46.50$ & 20.029 & 0.014 & 1.214 & 0.024 & 0.679 & 0.040 \\
\hline
\end{tabular}

${ }^{a}$ A portion of Table 2 is shown here for guidance regarding its form and content. Units of right ascensions are hours, minutes and seconds, and units of declination are degrees, arcminutes and arcseconds. Full Table 2 is only available in electronic form at the CDS via anonymous ftp to cdsarc.u-strasbg.fr (130.79.128.5) or via http://cdsweb.u-strasbg.fr/cgi-bin/qcat?J/A+A/418/33.

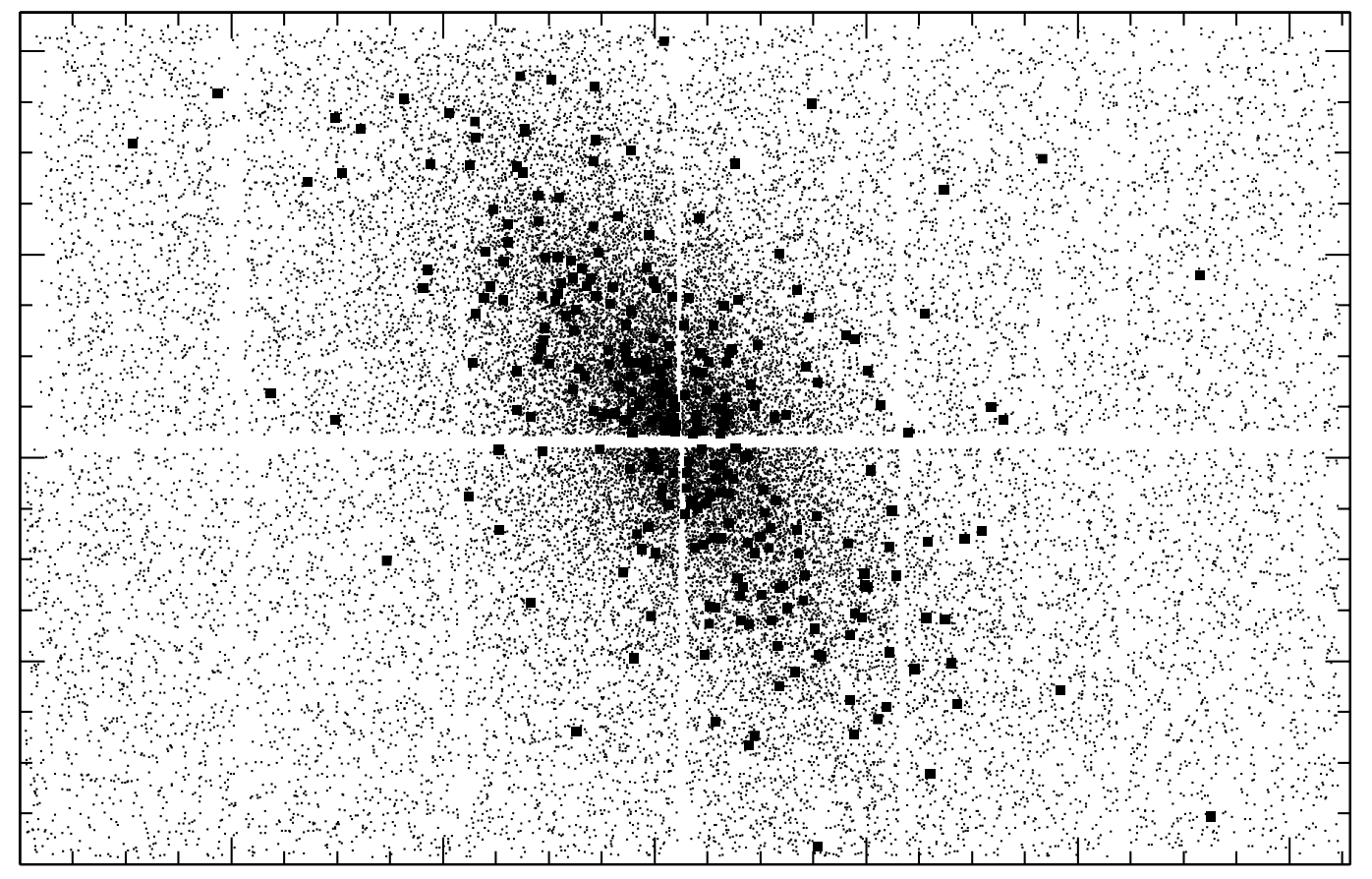

Fig. 3. Map of the CFH12K fields with all stars with good photometry. C stars are represented by large dots.

represented by a relatively narrow Gaussian (shown in Fig. 4). The average $I$ magnitude for the $288 \mathrm{C}$ stars is $\langle I\rangle=$ 20.31 with $\sigma_{I}=0.40$. If we adopt the true distance modulus $\mu_{0}=24.39$, derived by Han et al. (1997) from both HB and TRGB luminosities, the absolute average magnitude of $\mathrm{C}$ stars is then $\left\langle M_{I}\right\rangle=-4.39$, a value marginally lower than what is found in the other galaxies studied so far in the frame of our ongoing survey.

\subsubsection{Known C stars in NGC 147}

Recently, Nowotny et al. (2003) surveyed the C star population of NGC 147. Using essentially the same $\mathrm{CN}-\mathrm{TiO}$ technique than we employ, they identified $146 \mathrm{C}$ stars in a $6.5^{\prime} \times 6.5^{\prime}$ field centered on the galaxy. Since accurate coordinates of their stars are available at the CDS, we can compare our results.

A cross identification of their $\mathrm{C}$ star list with our database resulted in 109 matches (within one arcsecond), enough for a photometric comparison of the two data sets. We could, however, match their 146 stars by relaxing the coincidence criterion to $\sim 3^{\prime \prime}$ but then there are confusions on the matches. All these 109 matches do not, however, correspond to our C stars because Nowotny et al. (2003) adopt a different definition for $\mathrm{C}$ stars. Their blue limit is set at $(V-i)_{0}=1.16$, corresponding to $(R-I)_{0}=0.53$, quite bluer than our adopted limit of $(R-I)_{0}=$ 0.90 . This blueward shift introduces a number of fainter $C$ stars to the sample, as Demers \& Battinelli (2002) have shown. 


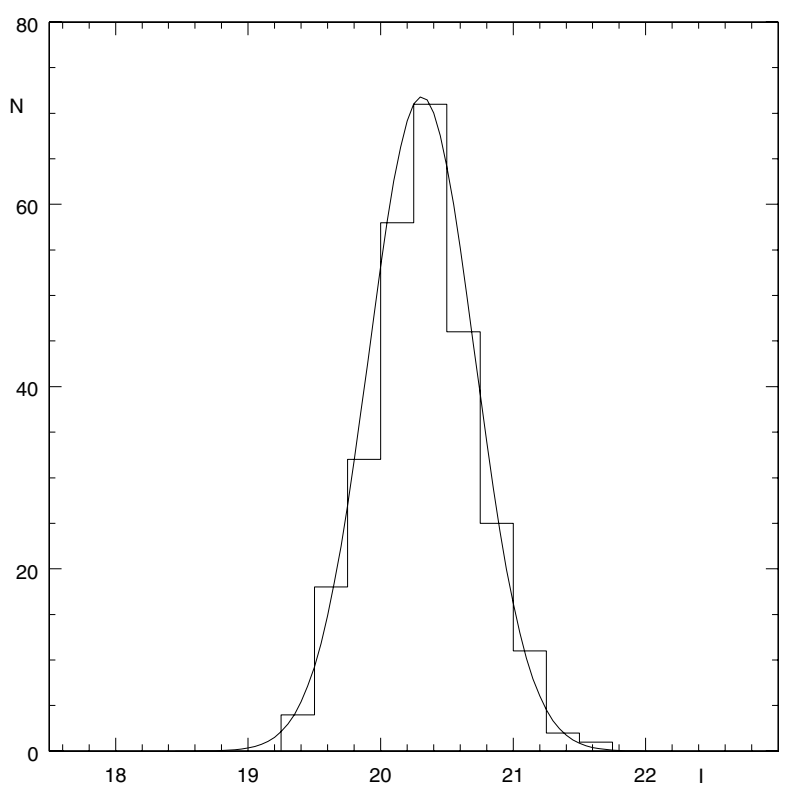

Fig. 4. Luminosity function of C stars in NGC 147.
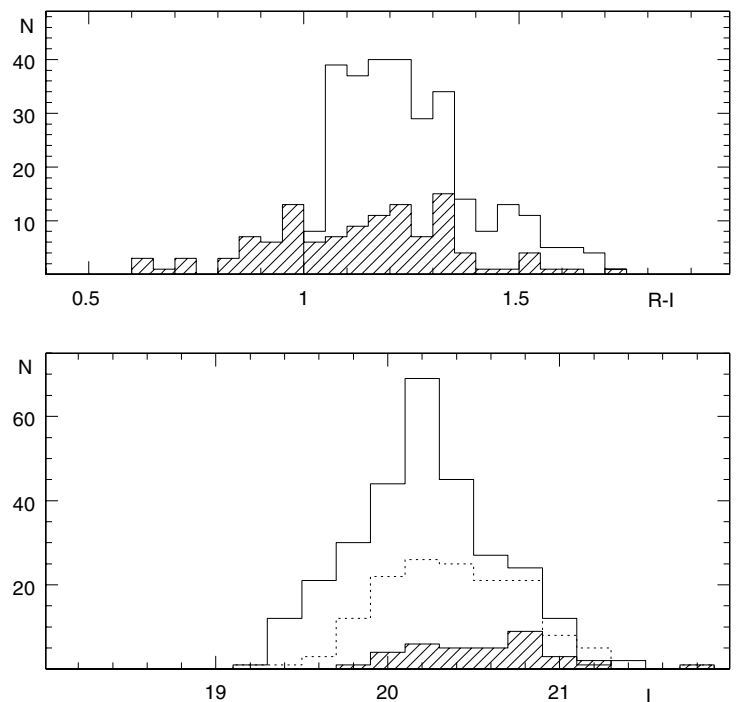

Fig. 5. Colour and magnitude comparisons between the $\mathrm{C}$ stars discovered in this survey and the ones identified by Nowotny et al. (2003). The upper panel compares the $(R-I)$ colour distributions revealing the different blue limits. The lower panel contrasts the luminosity functions. The dotted histogram is the Nowotny sample. The shaded area corresponds to their $\mathrm{C}$ stars with $(V-I)_{0}<1.75$, too blue to be included in our list.

The $(R-I)$ colour distribution of the 109 matches is compared to the colour distribution of our 288 sample, in the top panel of Fig. 5, to show the presence of a number of bluer C stars. The lower panel of Fig. 5 contrasts the LF of our $288 \mathrm{C}$ stars with their $146 \mathrm{C}$ stars (dotted histogram), taking into account their adopted extinction $A_{i}=0.336$. The shaded histogram represents their $\mathrm{C}$ stars with $(V-i)_{0}<1.75$, which is equivalent to $(R-I)_{0}<0.90$. Our apparent $I$ magnitudes are in reasonable agreement with their $i$ magnitudes, for such a crowded environment. For the 109 matched stars we calculate $\Delta_{I}=-0.26 \pm 0.12$, their apparent magnitudes being fainter.

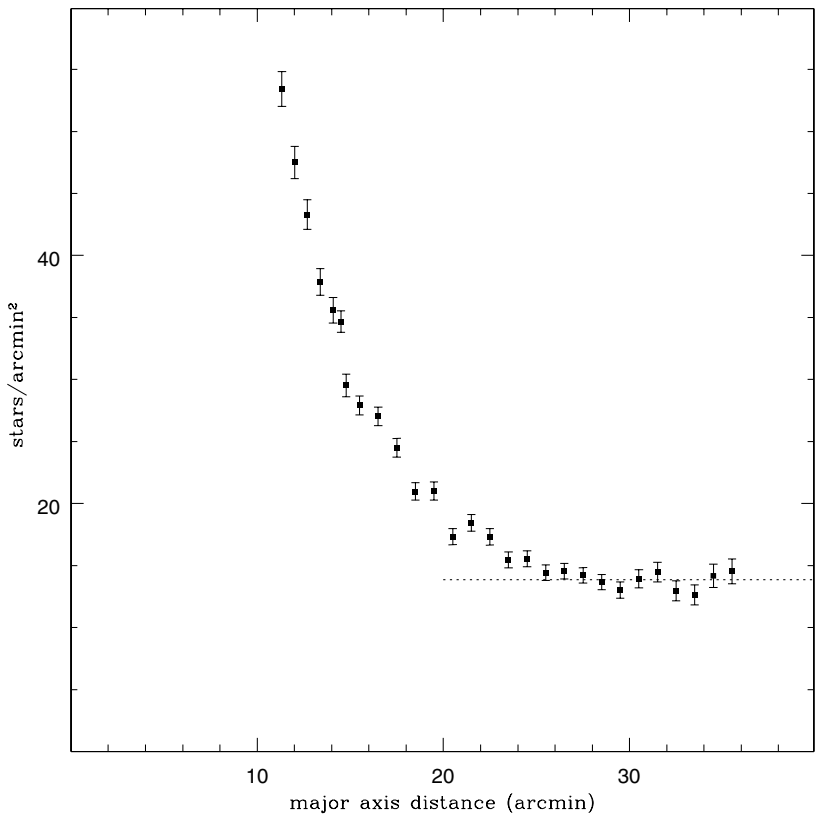

Fig. 6. Outer density profile of NGC 147 reaches a plateau at a major axis distance of $\sim 25^{\prime}$. The dashed line corresponds to the mean density of the last eleven points. We adopt this value for the foreground density toward NGC 147.

\subsection{The structure of NGC 147}

The structure of the spheroidal galaxy NGC 147 was first investigated by Hodge (1976). He determined that the position angle of the major axis is $34^{\circ}$ and that the ellipticity, even though increasing slightly from the center, reaches $\epsilon=0.44$ at $5^{\prime}$. Hodge fitted the luminosity profile of NGC 147 , up to $400^{\prime \prime}$ from the centre, to a King (1962) profile and deduced a tidal radius $r_{\mathrm{t}}=$ $10.1^{\prime}$, with a large uncertainty. Two years earlier, Kormendy \& Bahcall (1974) scanned photographs of NGC 147 and determined that the length of its major axis is $28.7^{\prime}$, implying that the tidal radius is certainly larger than the value quoted by Hodge. Kent (1987) attempted to measure the light profile of NGC 147 with CCD photometry but could barely reach $5^{\prime}$ on the major axis. He, however, showed that the profile is almost a pure exponential law with a scale length of $2.38^{\prime}$. Caldwell et al. (1992) re-measured the photographic profile of NGC 147 and obtained a scale length of $1.8^{\prime}$ with an ellipticity of $\epsilon=0.44$.

Having a $42^{\prime} \times 28^{\prime}$ mosaic centered on NGC 147 , we are in position to re-evaluate the profile and extent of NGC 147 . We employ for this exercise a file containing 54000 stars with good photometry $\left(\sigma_{(R-I)}<0.10\right)$ and reaching $I \approx 22.2$, the stars plotted in Fig. 1. We adopt Hodge's orientation of the major axis and also the ellipticity. We then count the stars in elliptical annulii centered on the galaxy. For major axis distances larger than $17^{\prime}$, ellipses become sufficiently large to spill outside of the CFH12K field, we then calculate the areas of the sectors of the ellipses inside of the field and use these number to evaluate the surface densities.

Figure 6 reveals that the enhancement belonging to NGC 147 can be seen up to a major axis distance of $\sim 25$ arcmin. It is worth noting that such major axis cutoff is not affected by the adopted photometric acceptance criterion 


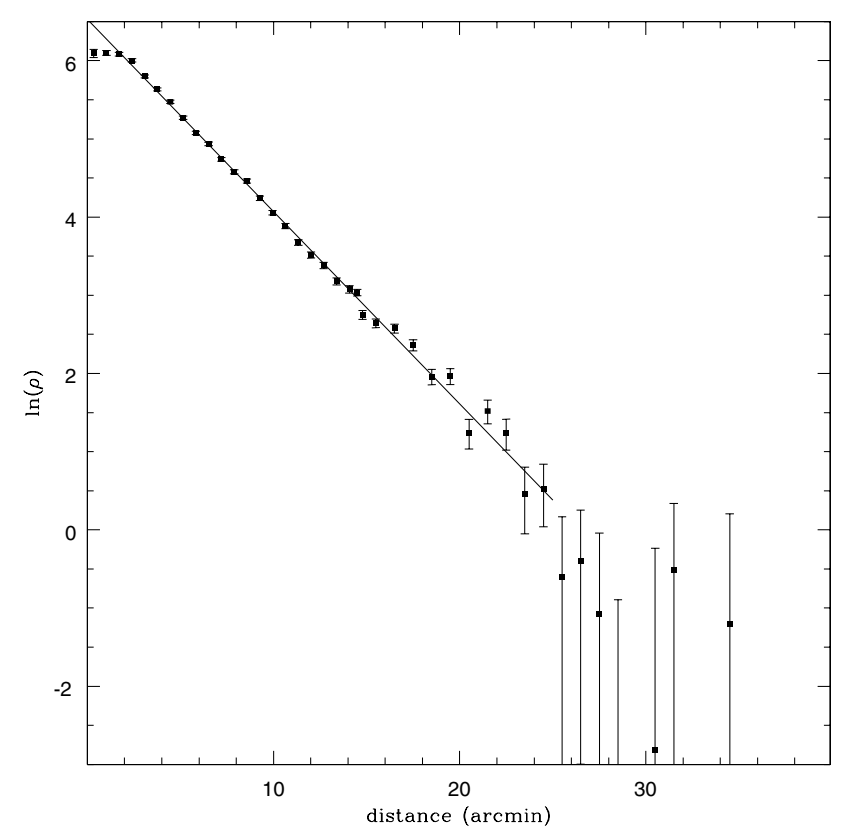

Fig. 7. The density profile of NGC 147 can be fitted to a power law with a scale length of $4.07^{\prime}$.

(see Sect. 3.1). Indeed, the relaxation of this criterion to $\sigma_{(R-I)}<0.20$ adds about 20000 faint stars (in the magnitude range $21.8<I<23.2$ ). Comparison of the density profiles with and without these faint stars reveals that count ratios for different annulii vary between 1.4 and 1.5 with no systematic radial trend. We therefore conclude that the error in colour is not strongly dependent on the crowding. From the last 11 points in Fig. 6, we estimate that the foreground contribution to the

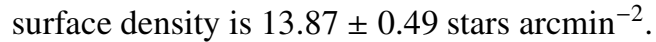

The foreground subtracted density profile of NGC 147, shown in Fig. 7, leads to a scale length of $4.1 \pm 0.1^{\prime}$, a value larger than all previous estimates, as described above.

Using the last points, above the foreground density, we can estimate a formal King's tidal radius from a plot of $\sqrt{\rho}$ versus $1 / r$. This relation, displayed in Fig. 8, yields $r_{\mathrm{t}}=33.9 \pm$ $2.4^{\prime}$. This implies that the halo of NGC 147 is much larger than previously believed. The presence of $\mathrm{C}$ stars far from the center of the galaxy confirms this finding.

The number of C stars in the periphery of NGC 147 is low but they do have the advantage over the giants to be seen behind a zero foreground. The density profile of $\mathrm{C}$ stars is shown in Fig. 9. The slope of the least square fit, equal to $-0.243 \pm$ 0.001 , is essentially identical ( $99 \%$ confidence limit) to the one in Fig. 7, and the resulting scale length is $4.1 \pm 0.3^{\prime}$.

This indicates that the intermediate-age population of NGC 147 is well mixed and has essentially the same extent than its old population. This population mixture appears to be a nearly universal property of dwarf galaxies massive enough to allow star formation during most of their life time. Indeed, our C star surveys of IC 1613 (Albert et al. 2000); NGC 6822 (Letarte et al. 2002); NGC 3109 (Demers et al. 2003) and WLM (in preparation) indicate that AGB stars and old stars have similar spatial distributions. NGC 185 is the only galaxy where the AGB population is obviously more concentrated than the old

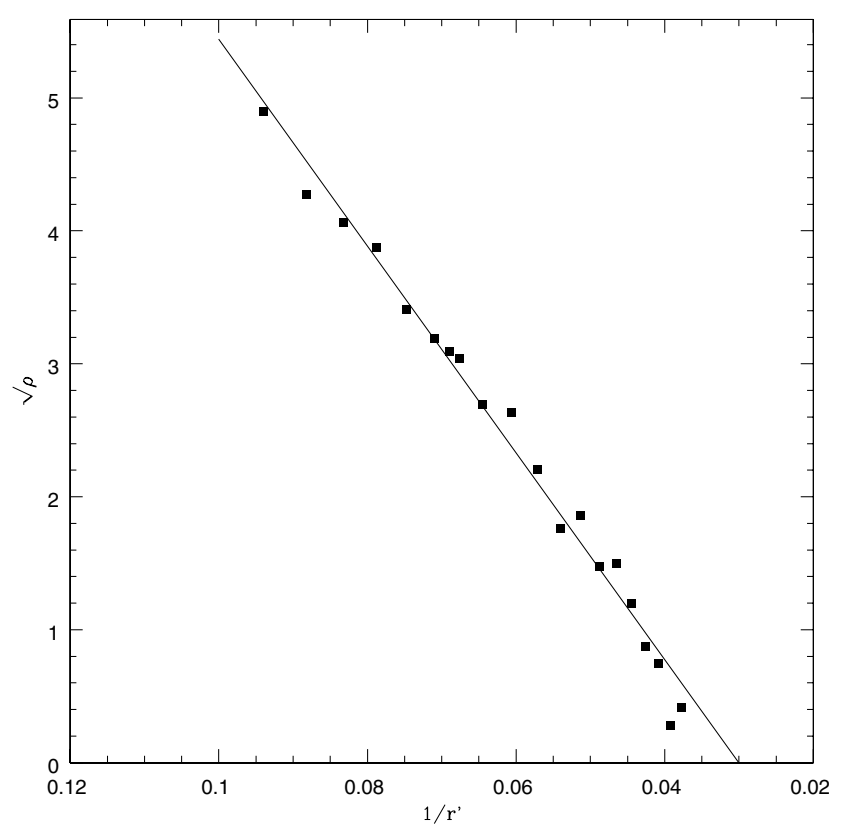

Fig. 8. The stellar density in the periphery of NGC 147 fits well the King relation to evaluate the tidal radius.

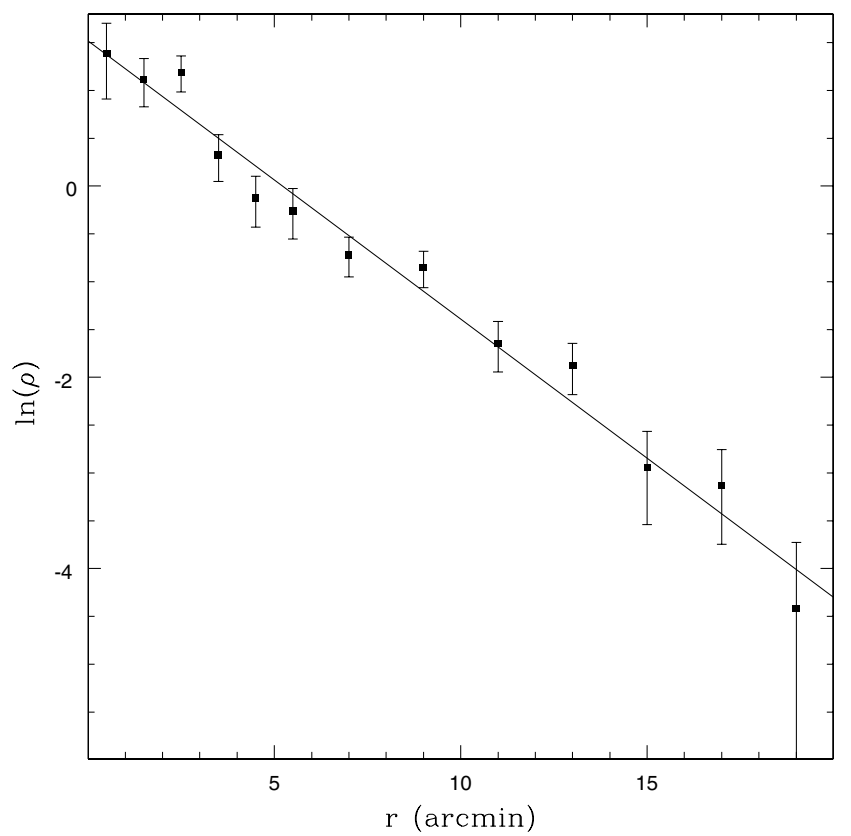

Fig. 9. The surface density profile of $\mathrm{C}$ stars fits the same power law than the old stellar population of NGC 147.

population (Battinelli \& Demers in preparation). C stars in the inner region of NGC 205 show an asymmetric distribution. Its proximity to M 31 makes it impossible to compare the distribution of its stellar populations in its outer parts. Both of these galaxies, contrary to NGC 147, have blue stars in their centre.

Using this scale length for $\mathrm{C}$ stars, one can estimate that there should be $\sim 5 \mathrm{C}$ stars outside of the ellipse of major axis of 20 arcmin. There are 7 in our CFH12K, thus very few NGC 147 C stars, if any, should be found outside of the surveyed area. 


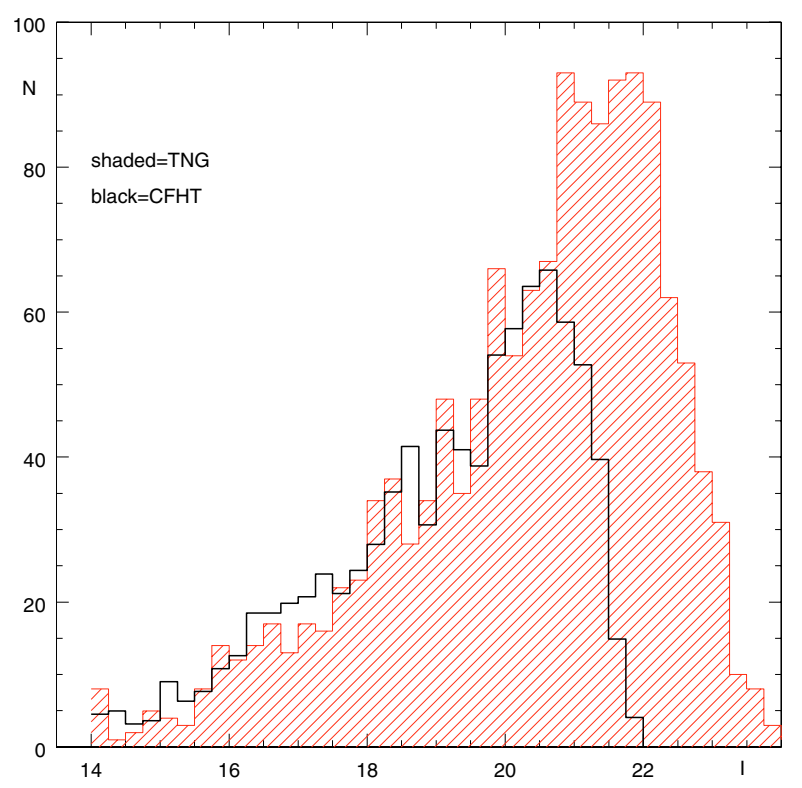

Fig. 10. Comparison of the foreground $I$-luminosity function for TNG and CFHT data.

\subsection{The $C / M$ ratio}

While stars in the C-rich box of the colour-colour diagram are uniquely AGB stars (there are no C-rich RGB stars), those in the O-rich box are a mixture of AGB and RGB stars. Thus, in order to deal exclusively with AGB stars (if, for example, we want to determine the $C / M$ ratio) it is necessary to discard those stars along the M-branch in the colour-colour diagram which belong to the Red Giant Branch. To select only AGB M stars we follow the procedure adopted by Battinelli et al. (2003) which consists in rejecting stars with $M_{\text {bol }}$ fainter than the TRGB luminosity as well as those brighter than the brightest members of the galaxy. The bright cutoff for the selection of AGB M stars is $I_{0}=18.48$ which corresponds to the value used by Battinelli et al. (2003) for M 31 scaled for the different distance and absorption of NGC 147. The application of these criteria yields some 3500 stars. Of course, not all of them are actually NGC 147 members and a careful correction for the foreground contamination is needed to get rid (statistically) of non-member stars. Since, our foreground-field photometry lacks of reliable narrow-band data (see Sect. 2), we select stars in two 1024 pixel wide strips located on the West and East sides of the CFHT mosaic. We are confident that these two strips well represent a foreground field since, as shown in Fig. 10, their $I$-luminosity functions are essentially identical (in the relevant magnitude range) to the one of the TNG foreground field. This is also confirmed by the behaviour at large distances of stellar counts along the major-axis of NGC 147 (see Sect. 4.2). To accomplish the statistical foreground subtraction we count how many stars satisfy the above criterion for the selection of AGB M stars in the two strips of the CFH12K field. We thus derive a foreground density of AGB M stars of $2.2 \pm$ $0.1 \operatorname{arcmin}^{-2}$ which leads to 2570 AGB M stars statistically belonging to NGC 147.

The overall ratio of $\mathrm{C}$-rich to O-rich AGB stars is then $C / M=0.24 \pm 0.02$ while its local values increases slightly with

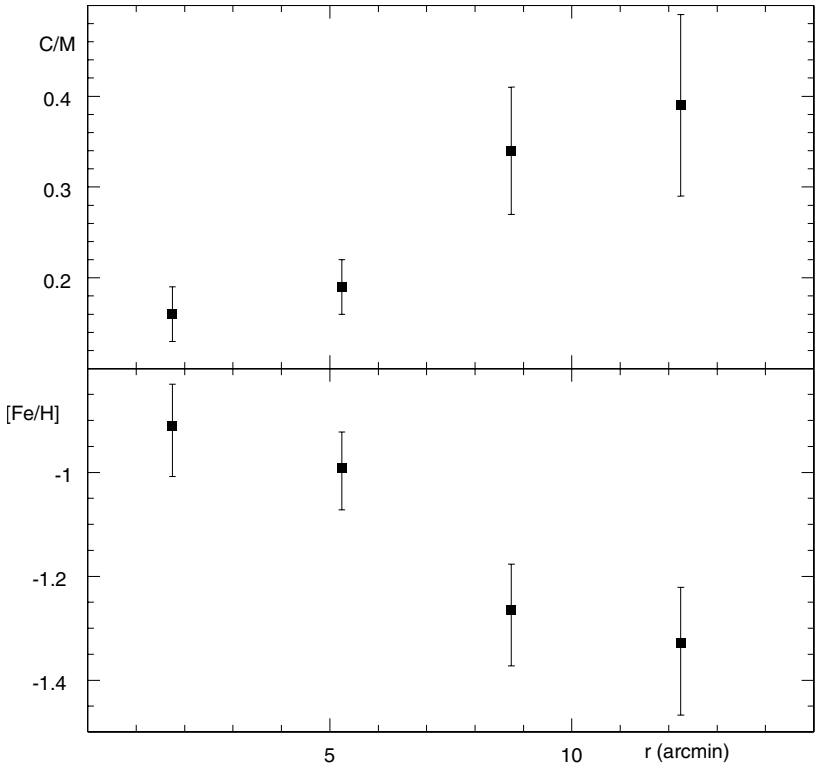

Fig. 11. Panel a): $C / M$ foreground corrected versus galactocentric distance; Panel b): corresponding $[\mathrm{Fe} / \mathrm{H}]$ radial gradient (see text).

the radial distance (see Fig. 11) in agreement with the metallicity radial gradient found by Han et al. (1997).

To convert $C / M$ ratios into $[\mathrm{Fe} / \mathrm{H}]$ it is in principle possible to adopt from the literature a relation like e.g. the Brewer et al. (1995) Eq. (3) obtained for the M 31 disk. However, we already noted (Battinelli et al. 2003) that many very red M stars were missed by Brewer et al. because these stars are too faint to be detected in the $V$-band, thus their calculated $C / M$ ratio is artificially increased. Very recently, Cioni \& Habing (2003) have published an analytical relation between the metallicity $([\mathrm{Fe} / \mathrm{H}])$ and the logarithm of the $C / M$ ratio by fitting data for several galaxies collected by Groenewegen (2002). Since, as explained by Cioni \& Habing (2003), it was not possible with the available data to determine the zero-point of such relation, we adopt their slope to derive the radial behaviour of $[\mathrm{Fe} / \mathrm{H}]$ shown in the lower panel of Fig. 6, fixing the zero point so to reproduce Han et al. (1997) central metallicity estimate. We conclude that $[\mathrm{Fe} / \mathrm{H}]$ of NGC 147 decreases by about $0.4 \mathrm{dex}$ over the plotted galactocentric distance range.

Acknowledgements. This research is funded in parts (S. D.) by the Natural Science and Engineering Council of Canada.

\section{References}

Albert, L., Demers, S., \& Kunkel, W. E. 2000, AJ, 119, 2780

Aparicio, A., \& Tikhonov, N. 2000, AJ, 119, 2183

Aparicio, A., Tikhonov, N., \& Karachentsev, I. 2000, AJ, 119, 177

Battinelli, P., Demers, S., \& Letarte, B. 2003, AJ, 125, 1298

Brewer, J. P., Richer, H. B., \& Crabtree, D. R. 1995, AJ, 109, 2480

Caldwell, N., Armandroff, T. E., Seitzer, P., \& Da Costa, G. S. 1992, AJ, 103, 840

Cioni, M.-R. L., \& Habing, H. J. 2003, A\&A, 402, 133

Cox, A. N. 2000, in Allen's Astrophysical Quantities, 4th ed. (New York: Springer), 392

Demers, S., \& Battinelli, P. 2002, AJ, 123, 238

Demers, S., Battinelli, P., \& Letarte, B. 2003, A\&A, 410, 795 
Groenewegen, M. A. T. 2002, Ringberg Castle Workshop Mouhcine, M. 2001, Ph.D. Thesis, Univ. L. Pasteur, Strasbourg [astro-ph/0208449]

Mouhcine, M., \& Lançon, A. 2003, MNRAS, 338, 572

Han, M., Hoessel, J. G., Gallagher, J. S. III, Holtzman, J., \& Stetson, P. B. 1997, AJ, 113, 1001

Hodge, P. W. 1976, AJ, 81, 25

Irwin, M. J. 1991, IAU Symp., 148, 453

Kent, P. C. II 1987, AJ, 94, 306

King, I. 1962, AJ, 67, 471

Kormendy, J., \& Bahcall, J. N. 1974, AJ, 79, 671

Landolt, A. U. 1992, AJ, 104, 340

Letarte, B., Demers, S., Battinelli, P., \& Kunkel, W. E. 2002, AJ, 123, 832

Lee, M. W., Freeman, W. L., \& Madore, B. F. 1993, AJ, 106, 964

Marigo, P. 2000, A\&A, 387, 507

Marigo, P., Girardi, L., \& Chiosi, C. 2003, A\&A, 403, 225

Martinez-Delgado, D., Aparicio, A., \& Gallart, C. 1999, AJ, 118, 2229

Mould, J. R., Kristian, J., \& Da Costa, G. S. 1983, ApJ, 270, 471

Nowotny, W., Kerschbaum, F., Olofsson, H., \& Schwarz, H. E. 2003, A\&A, 403, 93

Sage, L. J., Welch, G. A., \& Mitchell, G. F. 1998, ApJ, 507, 726 Saha, A., \& Hoessel, J. G. 1990, AJ, 99, 97

Saha, A., Hoessel, J. G., \& Mossman, A. E. 1990, AJ, 100, 108

Salaris, M., \& Cassisi, S. 1998, MNRAS, 289, 166

van den Bergh, S. 1998, AJ, 116, 1688

van den Bergh, S. 2000, The Galaxies of the Local Group, Cambridge Astrophysics Ser., 35

Weinberg, M. D., \& Nikolaev, S. 2001, ApJ, 508, 712

Young, L. M. 1999, ASP Conf. Ser., 163, 109

Young, L. M., \& Lo, K. Y. 1997, ApJ, 476, 127 